\title{
IL-9/IL-9 receptor signaling selectively protects cortical neurons against developmental apoptosis
}

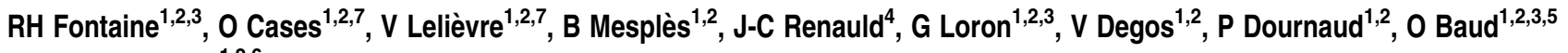 \\ and $\mathrm{P}$ Gressens $\mathbf{x}^{\star, 1,2,6}$
}

In mammals, programmed cell death (PCD) is a central event during brain development. Trophic factors have been shown to prevent PCD in postmitotic neurons. Similarly, cytokines have neurotrophic effects involving regulation of neuronal survival. Nevertheless, neuronal PCD is only partially understood and host determinants are incompletely defined. The present study provides evidence that the cytokine interleukin-9 (IL-9) and its receptor specifically control PCD of neurons in the murine newborn neocortex. IL-9 antiapoptotic action appeared to be time-restricted to early postnatal stages as both ligand and receptor transcripts were mostly expressed in neocortex between postnatal days 0 and 10 . This period corresponds to the physiological peak of apoptosis for postmitotic neurons in mouse neocortex. In vivo studies showed that IL-9/L-9 receptor pathway inhibits apoptosis in the newborn neocortex. Furthermore, in vitro studies demonstrated that IL-9 and its receptor are mainly expressed in neurons. IL-9 effects were mediated by the activation of the JAK/STAT (janus kinase/signal transducer and activator of transcription) pathway, whereas nuclear factor- $\kappa \mathrm{B}$ (NF- $\kappa \mathrm{B}$ ) or Erk pathways were not involved in mediating IL-9-induced inhibition of cell death. Finally, IL-9 reduced the expression of the mitochondrial pro-apoptotic factor Bax whereas Bcl-2 level was not significantly affected. Together, these data suggest that IL-9/L-9 receptor signaling pathway represents a novel endogenous antiapoptotic mechanism for cortical neurons by controlling JAK/STAT and Bax levels.

Cell Death and Differentiation (2008) 15, 1542-1552; doi:10.1038/cdd.2008.79; published online 13 June 2008

Programmed cell death (PCD) in the developing cerebral cortex is a major determinant in its formation. Approximately $50 \%$ of all cortical neurons generated are destined to die in two consecutive waves of PCD. The embryonic phase starts at embryonic day 14 (E14) and targets proliferating precursor cells, whereas a second postnatal phase targets postmitotic neurons from postnatal day (P)0 to P10 in the mouse. For both waves, cell death is apoptotic. ${ }^{1-4}$ Alteration of developmental cell death can be associated with the etiology of several human nervous system abnormalities including hydrocephaly and microcephaly. $1,5-7$

The mechanisms of PCD are remarkably conserved among species and the molecular machinery relies on the mitochondrial pathways of intracellular signal transduction., For example, null mutations of caspase-3 or caspase-9 in some mouse strains cause an enlarged forebrain due to the lack of normal neural progenitor apoptosis during development. ${ }^{10,11} \mathrm{Bax}$, one of the $\mathrm{BCl}-2$ family of genes, encodes a protein that regulates the rate at which cell populations undergo $P C D^{12}$ and promotes neuronal cell death during the development of the nervous system. ${ }^{13,14}$

To keep PCD of postmitotic neurons under strict control, trophic factors such as BDNF (brain-derived neurotrophic factor), NT4 (neurotrophin-4) or IGF-1 (insulin-like growth factor- 1$)^{15,16}$ may play a crucial role. Such factors may display not only pleiotropic roles but also a protracted expression from embryonic life to adulthood. The search for factors that display potent effects on developmental cortical PCD but with timerestricted and specific cellular expression patterns has, until now, remained unsuccessful, although this would be a major step toward understanding the regulation of brain development, as well as the ontogeny of certain development-related brain disorders.

The present paper provides the first evidence that the cytokine interleukin-9 (IL-9) is such a factor. This cytokine is chiefly known to target immune cells such as Th2 and B lymphocytes and mast cells. ${ }^{17} \mathrm{IL}-9$ actions are mediated through its interaction with its specific receptor (IL-9 receptor;

\footnotetext{
${ }^{1}$ Inserm, U676, Paris, France; ${ }^{2}$ Université Paris 7, Faculté de Médecine Denis Diderot, IFR02 and IFR25, Paris, France; ${ }^{3}$ Inserm, AVENIR, Paris, France; ${ }^{4}$ Ludwig Institute for Cancer Research, Brussels Branch, and Experimental Medicine Unit, Université de Louvain, Brussels, Belgium; ${ }^{5}$ AP HP, Hôpital Robert Debré, Service de Néonatologie, Paris, France and ${ }^{6}$ AP HP, Hôpital Robert Debré, Service de Neurologie Pédiatrique, Paris, France

${ }^{*}$ Corresponding author: P Gressens, INSERM U676, Hôpital Robert Debré, 48 blvd Serurier, F-75019 Paris, France.

Tel: + 33 (0) 14003 19 76; Fax: + 33 (0) 14003 19 95; E-mail: pierre.gressens @inserm.fr

${ }^{7}$ These authors have contributed equally to the present work

Keywords: IL-9; apoptosis; neurons; JAK/STAT; cytokines

Abbreviations: BCL3, B-cell CLL/lymphoma 3; BDNF, brain-derived neurotrophic factor; DIV, days in vitro; dl, deep layer; E, embryonic day; Fr, frontal cortex; GFAP, glial fibrillary acidic protein; HPRT, hypoxanthine-guanine phosphoribosyl-transferase; IGF-1, insulin-like growth factor-1; I $\kappa$ B, inhibitor of $\kappa \mathrm{B}$; IL, interleukin; IL-9R, interleukin-9 receptor; IL-9R KO, IL-9 receptor knockout mice; I.p., intraperitoneal; IRS, insulin receptor substrate; JAK/STAT, janus kinase/signal transducer and activator of transcription; M1, primary motor cotex; mMCP-1, mouse mast cell protease-1; NF- $\kappa$ B, nuclear factor- $\kappa$ B; NT4, neurotrophin-4; P, postnatal day; PBS, phosphate buffer saline; Parth, parthenolide; PCD, programmed cell death; PI3K, phosphatidyl-inositol 3-kinase; PKB, protein kinase B; S1, primary somatosensory cortex; Sp, septum; St, striatum; Stau, staurosporine; TGF- $\beta$, transforming growth factor- $\beta$; TCR- $\beta$, T-cell receptor- $\beta$; TUNEL, terminal transferase dUTP nick endlabeling; up, upper layer

Received 29.8.07; revised 28.4.08; accepted 05.5.08; Edited by M Deshmukh; published online 13.6.08
} 
IL-9R). ${ }^{18}$ IL-9/IL-9R signaling pathway mainly targets the downstream activation of JAK/STAT (janus kinase/signal transducer and activator of transcription) and subsequent phosphorylation cascades initiated by multiple kinases including IRS-PI3K-PKB (insulin receptor substrate, phosphatidyl-inositol 3-kinases, protein kinase-B) and Erk. ${ }^{17,19}$ IL-9 also seems to regulate NF- $\kappa \mathrm{B}$ (nuclear factor $-\kappa \mathrm{B}$ ) activity through BCL3 (B-cell CLL/lymphoma 3 ) gene induction that encodes a protein with close homology to $\mathrm{l} \kappa \mathrm{B}$ (inhibitor of $\kappa \mathrm{B}$ ) proteins. ${ }^{20}$ In mouse thymic lymphomas, these signaling pathways were recognized as critical to the antiapoptotic effect of IL-9. ${ }^{21}$ Effects of IL-9 on epithelial cells and neurons have also been described. ${ }^{17}$ For instance, IL-9 was shown to regulate the in vitro differentiation of hippocampal progenitor cells ${ }^{22}$ and human microglia-expressed mRNA transcripts for the specific IL-9R, ${ }^{23}$ but evidence for a possible physiological role of IL-9 in the developing brain remained uncertain when compared to other cytokines like IL- 6 or TGF- $\beta$ (transforming growth factor- $\beta$ ). ${ }^{24}$

The goal of the present study was to examine whether IL-9 plays a role during neuronal PCD. We demonstrated for the first time that not only does the expression of the IL-9/IL-9R system peak during the time frame of the second wave of $P C D$ but also that IL-9-mediated apoptosis is a central determinant of brain development. This effect is mediated through the JAK/ STAT pathway and the reduced expression of the mitochondrial pro-apoptotic factor Bax.

\section{Results}

IL-9 and IL-9R expressions peak between P0 and P10 in the cerebral neocortex. Quantitative real-time PCR performed on total mRNA extracted from neocortex of Swiss mice between E14 and P60 showed a sharp increase of $I L-9 R$ mRNA in the early postnatal period with a peak at P5 followed by a decrease to baseline by P20 (Figure 1a). IL-9R belongs to the hematopoietic cytokine receptor superfamily and consists of dimers combining one specific IL-9R $\alpha$-chain and one $\gamma$-chain shared by IL-2, $-4,-7$ and -15 receptors. ${ }^{18}$ The presence of this $\gamma$-chain was also detected in P5 mouse brain, supporting the functionality of the IL-9R at this age (Figure 1a, inset 1).

Similarly, quantitative real-time PCR showed a peak expression of IL-9 mRNA between P0 and P10 (Figure 1b).

Using DIG-labeled riboprobes encoding the full length of murine IL-9R, we determined the spatial-temporal pattern of IL-9R mRNA in the developing telencephalon. During embryonic development, we did not detect $I L-9 R$ mRNA in the cerebral cortex although there was a weak signal in the septum. By P1, strong levels of $I L-9 R$ mRNA were present in the deep layers $(\mathrm{V}-\mathrm{VI})$ of the primary motor, somatosensory and frontal cortices (Figure 1c). Low levels of IL-9R mRNA were also detected in the upper layers (II-IV) of the cortex. By P5, IL-9R mRNA was observed in all cortical layers, but especially in layers II-III and VI (Figure 1c). By P20, IL-9R mRNA expression was no longer detectable in any telencephalic structure.

These data show that $I L-9 / I L-9 R$ mRNAs have a peak of expression between $\mathrm{P} 0$ and $\mathrm{P} 10$ in the mouse neocortex, a period corresponding to the peak of PCD of neocortical postmitotic neurons. ${ }^{3,4}$

IL-9 and IL-9R are predominantly expressed in neurons. In vitro experiments showed a predominant expression of $I L-9$ and IL-9R mRNAs in neocortical neurons, whereas the expression of these transcripts remained low in astrocytes and non-detectable in oligodendrocytes and microglia. Expression of IL-9R mRNA in neurons was comparable to the level observed in mast cells, whereas the expression of IL-9 mRNA was roughly fourfold higher in mast cells when compared to neurons (Figure $1 \mathrm{~d}-\mathrm{e}$ ). $\gamma$-chain mRNA was also detected in primary neuronal cultures, which supports the functionality of the IL-9R in this cell type (Figure 1d, inset).

These in vivo and in vitro findings, together with evidence that IL-9 is known to regulate T-lymphocyte apoptosis, ${ }^{21}$ led us to investigate the potential role of IL- 9 and its receptor in neocortical neuronal PCD between $\mathrm{P0}$ and $\mathrm{P} 10$.

Effects of IL-9 on neocortical neuronal apoptosis. As PCD of neocortical postmitotic neurons peaks at P4-P6 in mice ${ }^{4}$ the effect of IL-9 on neuronal apoptosis was studied using P5 IL-9R knockout (IL-9R KO) mice C57BI6 pups and IL-9-treated P5 Swiss pups.

In IL-9R KO mice, the density of cleaved caspase-3-positive cells was increased two- to fourfold in the superficial layers (layers II-III) of motor, somatosensory and visual neocortical areas when compared to wild-type control mice (Figure 2a and b). Intraperitoneal (i.p.) injections of IL-9 from P1 to P5 in IL-9R KO mice did not significantly affect levels of apoptosis when compared to untreated IL-9R KO mice (Figure $2 b$ ). This supports the hypothesis of an endogenous antiapoptosis function of IL-9 in developing mouse cortex, through a direct effect of IL-9 on its receptor. To confirm this result, we explored the role of i.p. administration of exogenous IL-9 in mouse pups from P1 to P5. This experimental procedure resulted in a twofold reduction in cleaved caspase-3 staining of cerebral sections in the different cortex area (Figure $2 \mathrm{c}$ and $\mathrm{d}$ ). Thus, repeated i.p. injection of IL-9 during the specific time window of peak expression of endogenous IL-9R induced a selective antiapoptotic effect on neocortical neurons in vivo at P5, corroborating data obtained using IL-9R KO mice. Similar results were obtained with terminal transferase dUTP nick end-labeling (TUNEL) staining (Supplementary Figure 1) in IL-9-treated Swiss pups.

We performed double immunohistochemistry experiments, combining anticleaved caspase- 3 and neural markers to demonstrate in vivo that dying cells in the neocortex are neurons (Figure 3). Typically, dying cells displayed mainly a pyramidal shape as detected with anticleaved caspase-3 staining (Figure 3a). In addition, anticleaved caspase-3 staining was largely colocalized with Brn-1, a transcription factor selectively expressed by glutamatergic neurons (Figure $3 d$ and e). In contrast, we did not observe any colocalization between anticleaved caspase-3 staining and anti-glial fibrillary acidic protein (anti-GFAP; an astrocytic marker) staining or somatostatin (a marker of a restricted subpopulation of interneurons) staining (Figure $3 \mathrm{~b}$ and $\mathrm{c}$ ). 

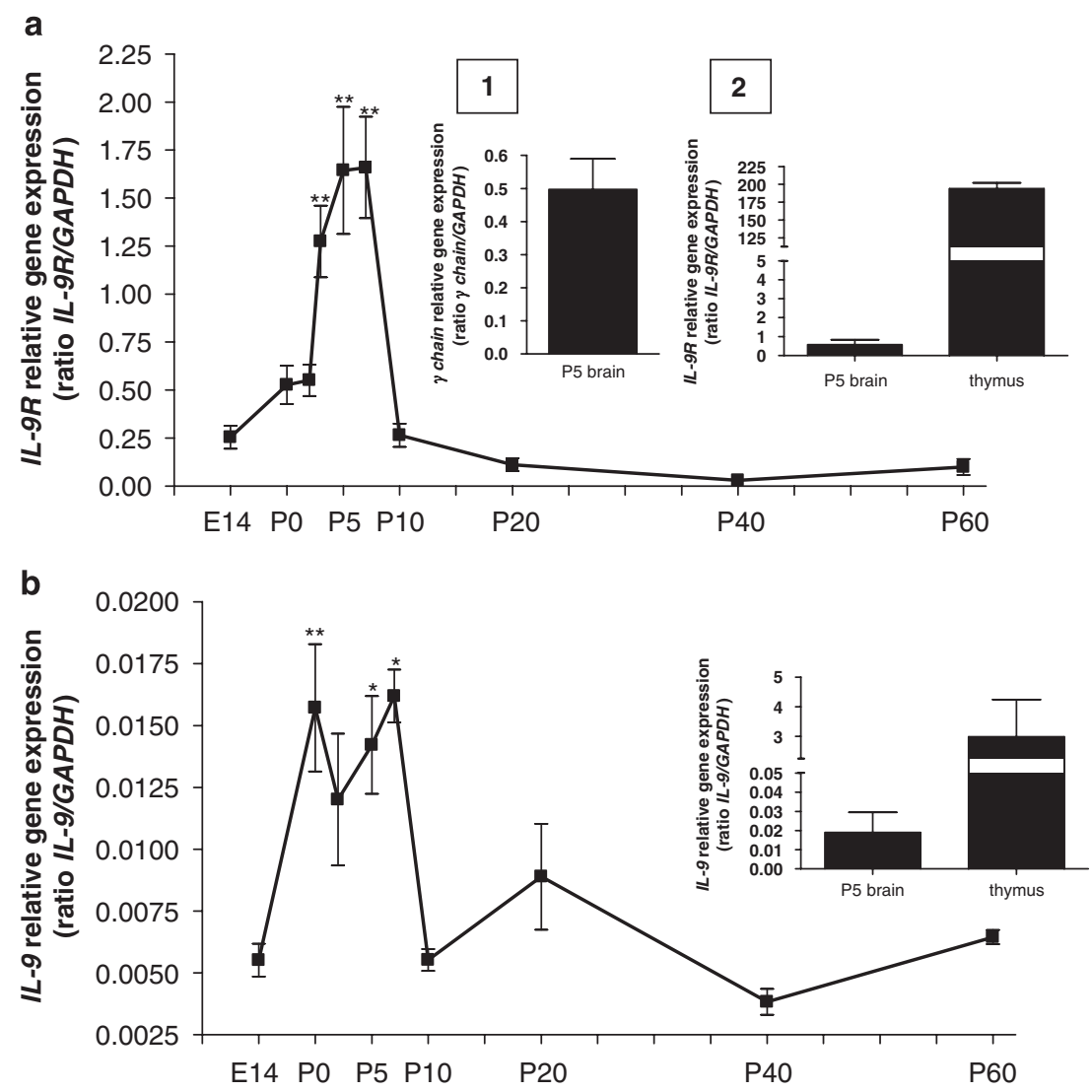

c

P5

P20
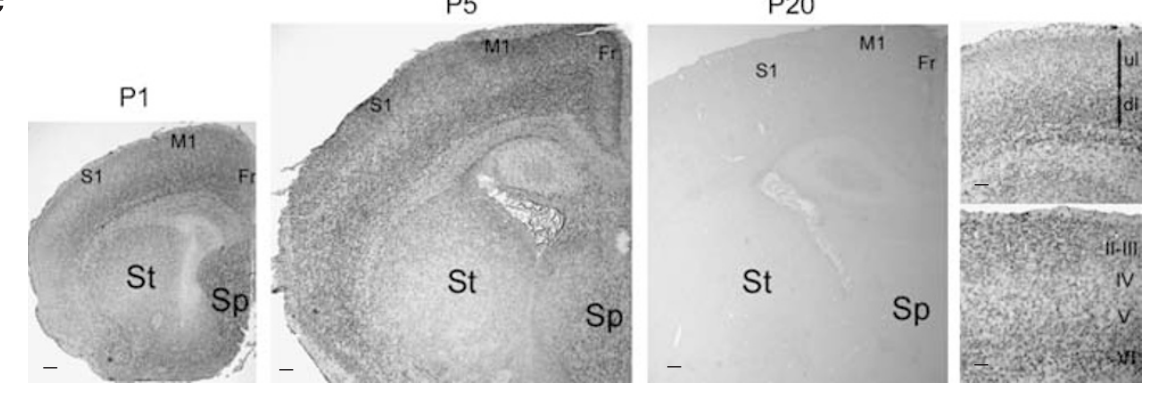

P1
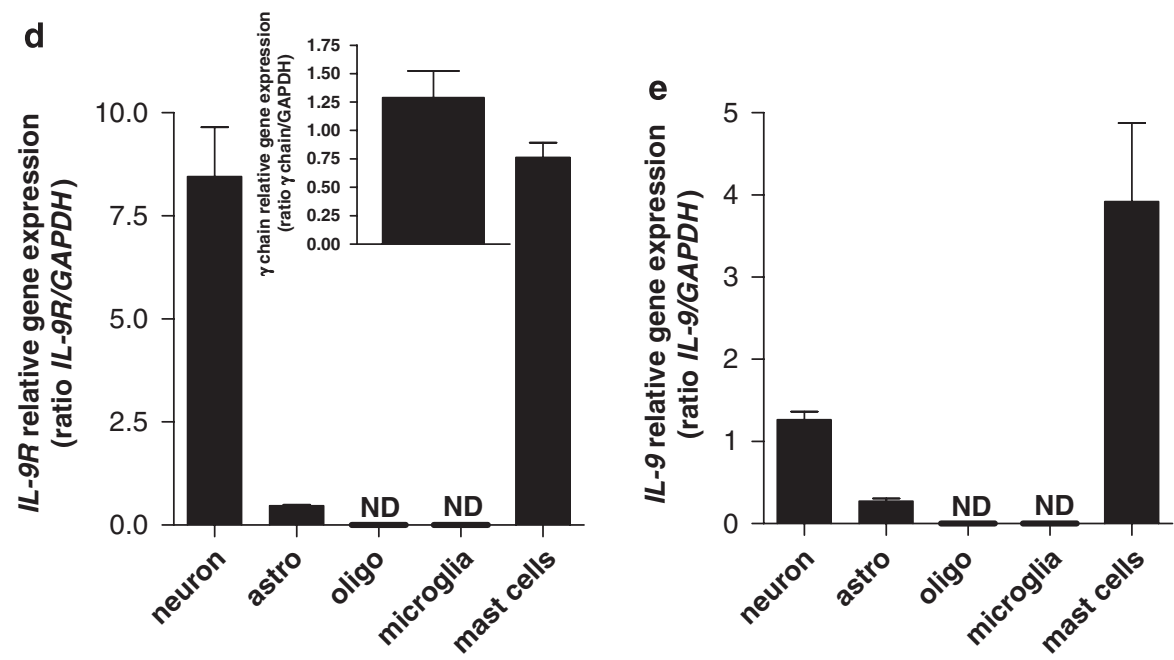
We tested in vitro the effects of a 200 U IL-9 treatment on primary neocortical neuronal cultures between DIV (days in vitro) 5 and 7 . In this model, IL-9 induced a significant decrease in the number of dying cells as quantified by Hoechst staining (Figure $4 a$ and b). Similarly, IL-9 treatment induced a significant decrease in the number of cleaved caspase-3-expressing cells (Figure 4c). Conversely, using the MTS/Formazan assay, IL-9 treatment significantly increased the cell viability (Figure 4d). In contrast, IL-9 treatment had no effect on the percentage of picnotic nuclei in IL-9R KOcultured neurons (Figure 4e). No effects were observed in neurons treated with IL-9 between DIV 10 and 12 or between DIV 12 and 14 (Figure 4b). This latter result was clearly correlated with quantitative PCR data showing higher expression levels of IL-9R in DIV 7 neurons, followed by a marked reduction of its expression in DIV 12 or DIV 14 neurons (Figure 4e). Finally, staurosporine (a protein kinase inhibitor with pro-apoptotic effects) displayed a dosedependent stimulation of cell death that was significantly reduced by cotreatment with IL-9 (Figure 4f), further supporting the antiapoptotic effect of IL-9 on neocortical neurons.

Cytoarchitectonics and neuronal density at adulthood in mice treated with IL-9R KO mice. The macroscopic examination of adult IL-9R KO mouse brain did not reveal any detectable abnormality when compared to agematched wild-type controls (Supplementary Figure 3a). Microscopic examination showed a preserved neocortical cytoarchitectonics and a normal density of NeuN-positive neurons when compared to controls (Supplementary Figure $3 b)$. Additional studies will be necessary to fully characterize the neuronal phenotype of the adult IL-9R KO mice.

Transduction pathways involved in IL-9 antiapoptotic effects on neocortical neurons. Cultured neurons were treated with pharmacological inhibitors in the presence or in the absence of IL- 9 treatment to determine the upstream effectors of IL-9-targeted caspase-3 activation. The results were supported by western blot analysis.

We addressed the hypothesis of IL-9-dependent NF- $\kappa$ B or Erk pathway activation using parthenolide and PD98059 inhibitors, respectively. Neither parthenolide nor PD98059 affected IL-9-induced cell death reduction when tested at pharmacological concentrations (Figure $5 a$ and b). Levels of phosphorylated forms of NF- $\kappa \mathrm{B} / \mathrm{l}-\kappa \mathrm{B}$ as well as of phosphoErk1/2 detected by western blots were not affected by IL-9 treatment, respectively (insets, Figure $5 a$ and $b$ ). These data demonstrate that NF- $\kappa$ B or Erk pathways were not involved in mediating IL-9-induced inhibition of cell death.

We tested the JAK/STAT pathway inhibitor AG490 in similar conditions (Figure 5c). IL-9 antiapoptotic effects were significantly and dose-dependently reversed by AG490 (Figure 5c). These data suggest that IL-9 antiapoptotic effects require JAK/STAT activation. This hypothesis was further confirmed in western blots, which show that IL-9 treatment induces phosphorylation of STAT proteins 1 and 3 (Figure $5 d$ and e, insets). IL-9 did not modify the concentrations of total STAT proteins 1 and 3 (Supplementary Figure 2).

The Bcl-2 family protein can be regulated by the JAK/STAT pathway. ${ }^{25} \mathrm{IL}-9$ triggers the reduction of Bax levels (Figure $5 \mathrm{f}$, inset), whereas $\mathrm{Bcl}-2$ or $\mathrm{Bcl}-\mathrm{xl}$ (Figure $5 \mathrm{~g}$ and $\mathrm{h}$, insets) were not significantly affected by IL- 9 treatment. These data strongly support the conclusion that IL-9-mediated antiapoptotic effect is produced via the JAK/STAT pathway (especially phosphorylation of STAT1 and 3) and the downregulation of Bax, a well-known pro-apoptotic factor influencing brain development. ${ }^{13,14}$

\section{Discussion}

IL-9R and its ligand IL-9 displayed a peak of expression between P0 and P10 in the mouse neocortex, an ontogenic period corresponding to the peak of PCD of neocortical postmitotic neurons. ${ }^{5}$ In agreement with this time-restricted expression, the IL-9/IL-9R signaling pathway significantly prevented neuronal PCD both in vivo and in vitro. This antiapoptotic effect seemed to similarly affect different cortical areas (motor, somatosensory and visual cortices) but, within each cortical area, to preferentially affect the superficial layers as supported by anticleaved caspase- 3 immunostaining and in situ hybridization for IL-9R mRNA. These data expand our knowledge about factors controlling PCD of postmitotic neocortical neurons, identifying a non-classical Th2 cytokine as a new antiapoptotic molecule. This antiapoptotic effect of the IL-9/IL-9R signaling pathway seemed highly specific as the expression of IL-9R and its ligand was highly restricted to the period of PCD in the developing murine neocortex.

\footnotetext{
Figure 1 Ontogenic pattern of $I L-9$ and $I L-9 R$ mRNA during murine neocortical development. (a and $\mathbf{b})$ Quantification of $I L-9 R$ (a) and $I L-9$ (b) mRNA in mouse neocortex between $\mathrm{E} 14$ and $\mathrm{P} 60$ by quantitative real-time PCR using total RNA samples isolated from Swiss mice ( $n=6 /$ group/age). The time-course analysis showed a significant peak between P0 and P10 for both the genes. Results are expressed as mean of the specific ratio \pm S.E.M. calculated as the relative expression level of IL-9 or IL-9R/GAPDH. (a, inset 1-2) Quantification of the $\gamma$-chain in P5 Swiss mice (a1) and relative expression of IL-9R in P5 brain and thymus, a major source for IL-9R (a2). (b, inset) Relative expression of $I L-9$ in P5 brain and thymus, a major source for IL-9. (c) Distribution of the IL-9R in the mouse neocortex visualized by in situ hybridization. Coronal sections (10 $\mu \mathrm{m}$ thick) from three different animals at P1, P5 or P20, respectively, were prepared on cryostat. DI, deep layers; Fr, frontal cortex; M1, primary motor cortex; S1, primary somatosensory cortex; Sp, septum; St, striatum; ul, upper layers. Scale bar $=50 \mu \mathrm{m}$ for P1, P5 and P20, scale bar $=25 \mu \mathrm{m}$ for P1 and P5 for microphotographs at higher magnification. (d) Expression levels of both IL-9 receptor and ligand were mainly found on neuronal and mast cell populations. Highly purified cultures of neurons at DIV 7 , astrocytes, oligodendrocytes, microglia and mast cells were tested for IL-9 and IL-9R expression by quantitative real-time PCR following a similar protocol as the one used for in vivo samples. Although cultured neurons and mast cells expressed significant levels of $I L-9$ and IL-9R transcripts, astrocytes displayed a very limited level of RNA encoding the ligand and its receptor. No amplification was obtained in similar conditions with RNA extracted from oligodendrocyte precursors and microglia (ND, non detectable). Data are presented as mean \pm S.E.M. of $I L-9$ or $I L-9 R / G A P D H$ ratio. Each experiment was run twice using independent cultures, and in both cases, samples were assessed in triplicate. One-way ANOVA followed by post hoc test (time-course analysis) showed significant differences at ${ }^{\star *} P<0.01$ or ${ }^{\star} P<0.05$. (d, inset) $\gamma$-chain mRNA was detected in primary neuronal cultures using the same protocol
} 
The evidence that IL-9 acts on neurons is consistent with original findings by Mehler et al., ${ }^{22}$ showing a regulation of neuronal differentiation of immortalized murine hippocampal progenitor cells mediated by IL-9 and with a previous study showing that IL-9 upregulates TGF- $\beta 1$ expression in developing mouse neurons both in vivo and in vitro. ${ }^{26}$

a
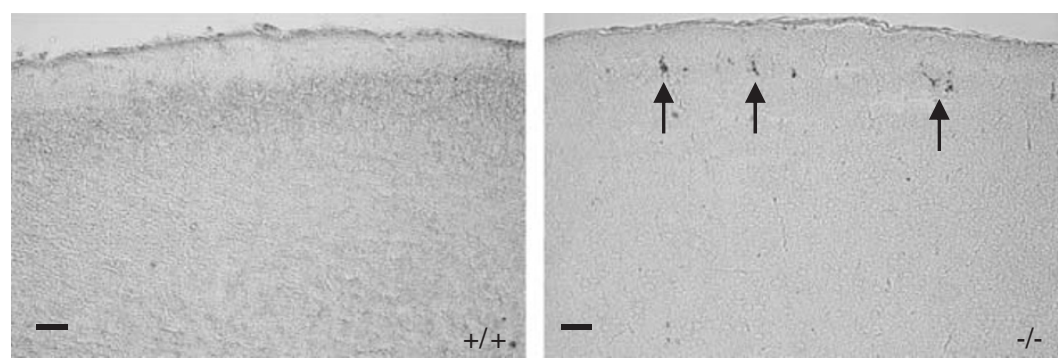

b

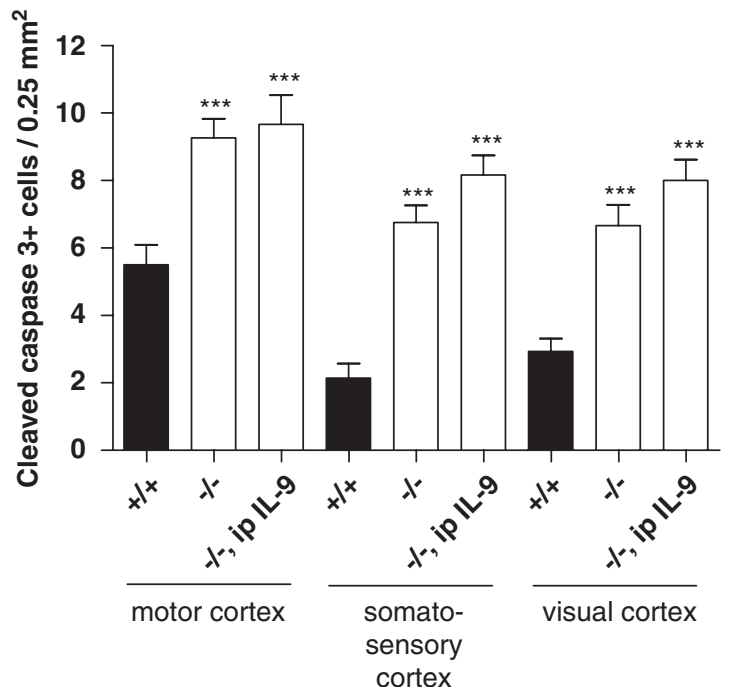

c

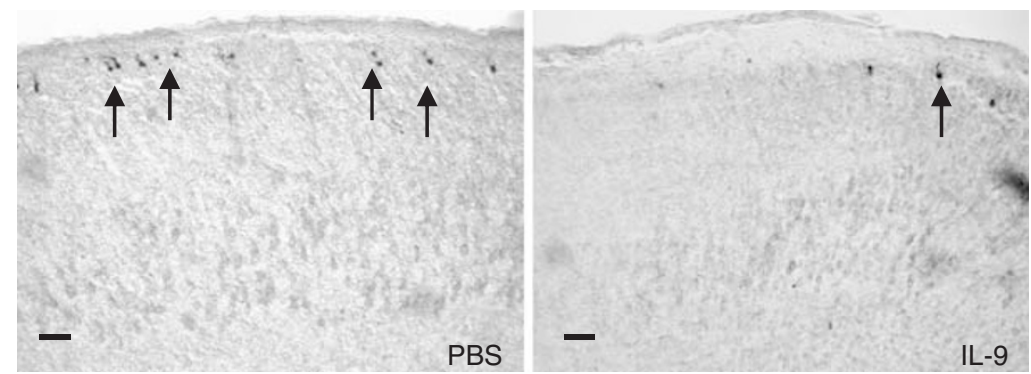

d

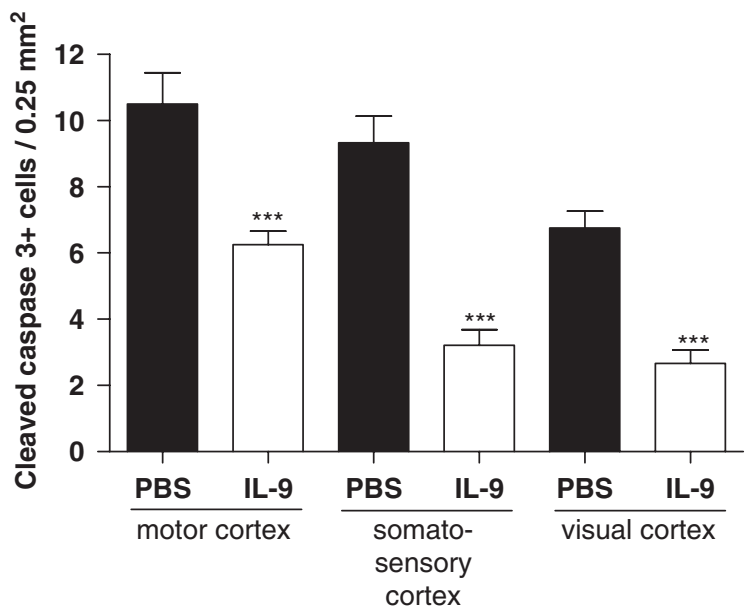


In the present study, the detection of mRNA for both $I L-9$ and $I L-9 R$ in neurons suggests the existence of an IL-9mediated autocrine and/or paracrine loop that can regulate neuronal PCD during development.
One study using PCR has previously reported the expression of IL-9R in human microglial cells prepared from embryonic telencephalon tissues. ${ }^{23}$ whereas we were unable to reproduce these data in mouse microglia. This discrepancy
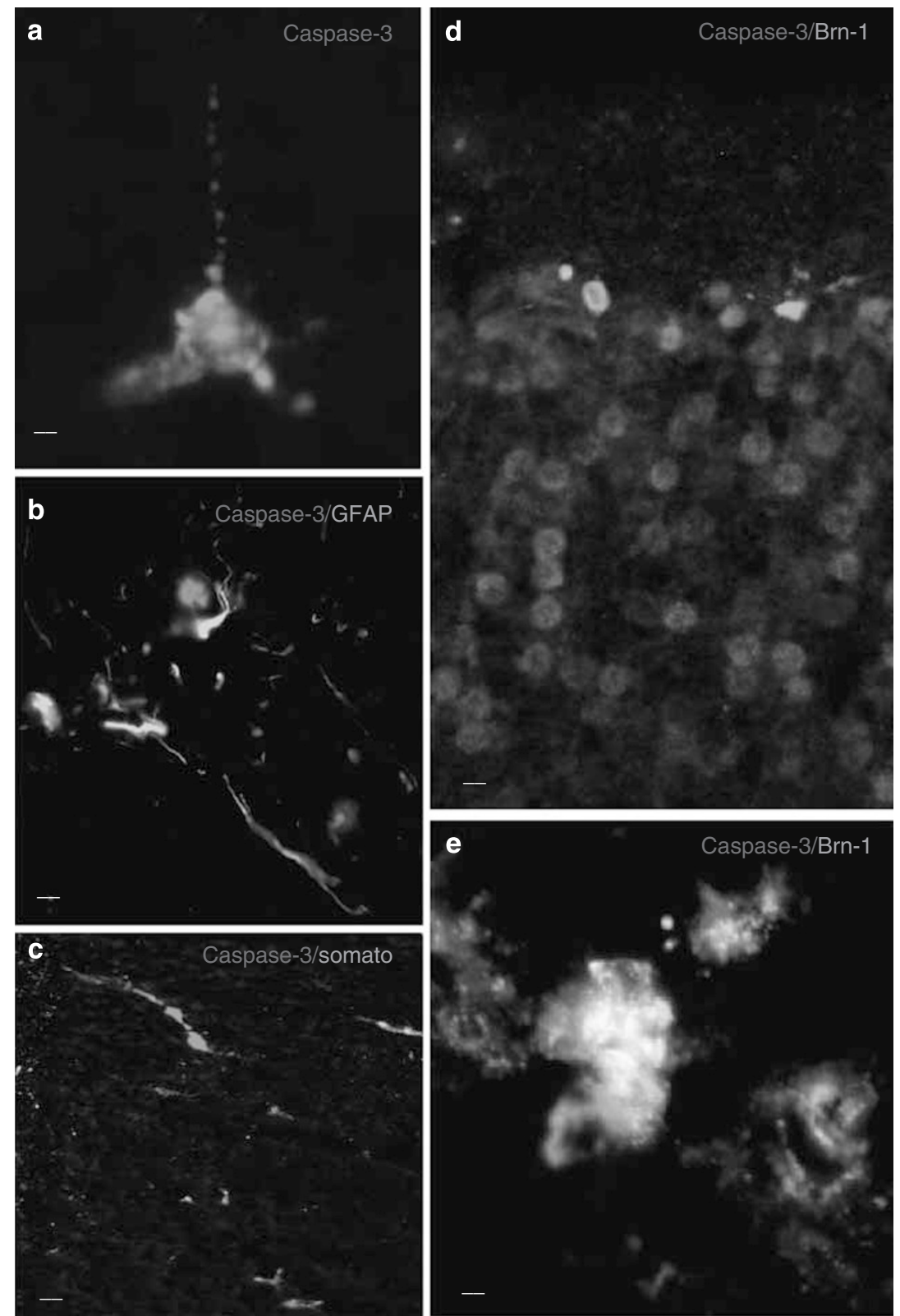

Figure 3 Phenotype of dying cells (recognized by anticleaved caspase-3 staining) in untreated P5 Swiss mice at P5. (a) In layer III of the cerebral cortex, cleavage of caspase-3 was only observed in large pyramidal neurons. Scale bar $=10 \mu \mathrm{m}$. (b and c) Double immunohistochemistry showing that anticleaved caspase-3 staining (red) is not colocalized with anti-GFAP or anti-somatostatin staining (green). Scale bar $=20 \mu \mathrm{m}$. (d and e) Double immunohistochemistry in layer II of S1 showing that anticleaved caspase-3 staining (red) is colocalized with anti-Brn1 staining (green). Scale bar $=20 \mu \mathrm{m}$ for microphotograph $\mathrm{d}$ and scale bar $=10 \mu \mathrm{m}$ for microphotograph $\mathbf{e}$

\footnotetext{
Figure 2 Antiapoptotic effect of IL-9 i.p. injection on mouse cortex is lost in IL-9R KO mice. (a and b) IL-9R KO (-/-) mice showed increase in physiological cell death compared to wild type $(+I+)$, as shown by anticleaved caspase-3 staining (microphotographs (a), arrows point to cleaved caspase-3-positive cells). Cleaved caspase-3positive cell quantification (b) showed that KO mice present an overall significant increase in numbers of dying cells at P5. I.p. injection of IL-9 from P1 to P5 in IL-9R KO ( $-/-$, i.p. IL-9) mice did not significantly affect cell death when compared to control IL-9R KO mice. (c and d) I.p. injection of IL-9 induced opposite results compared to KO mice. Activated caspase-3 was greatly reduced in P5 mouse cortices in IL-9 i.p. injected animal (IL-9) when compared to PBS-injected control animals (PBS; microphotographs (c), arrows point to cleaved caspase-3-positive cells). Quantification of cleaved caspase-3-positive cells (d) confirmed the antiapoptotic effect of i.p. injections of IL-9. Quantification was done under double-blinded procedure on four different fields specifically localized within the different cortical areas on adjacent sections performed using paraffin-embedded brain isolated from six different animals per group. $T$-test statistical analysis showed significant differences at ${ }^{* \star *} P<0.001$. Scale bar $=50 \mu \mathrm{m}$
} 
could relate to species differences. It is worth noting that in this study looking at human microglia, the significance of their results is unclear as the size of IL-9R PCR amplicon determined by gel electrophoresis did not correspond to the expected size calculated from their primer sequences. From a technical point of view, we cannot exclude that, in the present study, the absence (oligodendrocyte and microglia) or very low level (astrocytes) of mRNA for $I L-9 R$ in glial cells is due to the relatively long maintenance ( 3 weeks) of these cells in culture before the assay when compared to cultured neurons (7 days).

In our study, the presence of mast cells in primary neuronal cell culture was excluded by showing the lack of detectable
mRNA for the specific mouse mast cell protease-1 (mMCP-1) by PCR (data not shown). Similarly, the presence of lymphocytes in primary neuronal cell culture was excluded by showing the lack of detectable mRNA for T-cell receptor- $\beta$ (TCR- $\beta$ ) chain (data not shown). As IL-9R is known to be expressed by mast cells and as mast cells are present in the brain especially during development, ${ }^{26}$ we cannot exclude a role of brain mast cells in the in vivo antiapoptotic effects of IL-9. IL-9 antiapoptotic effects were mediated by the downstream JAK/STAT pathway and in particular STAT1 and STAT3, two of the major transcription factors previously shown to transduce IL-9 effects and mediate antiapoptotic effects of IL-9 in a T-lymphocyte cell line. ${ }^{27}$ Although IL-9 was a
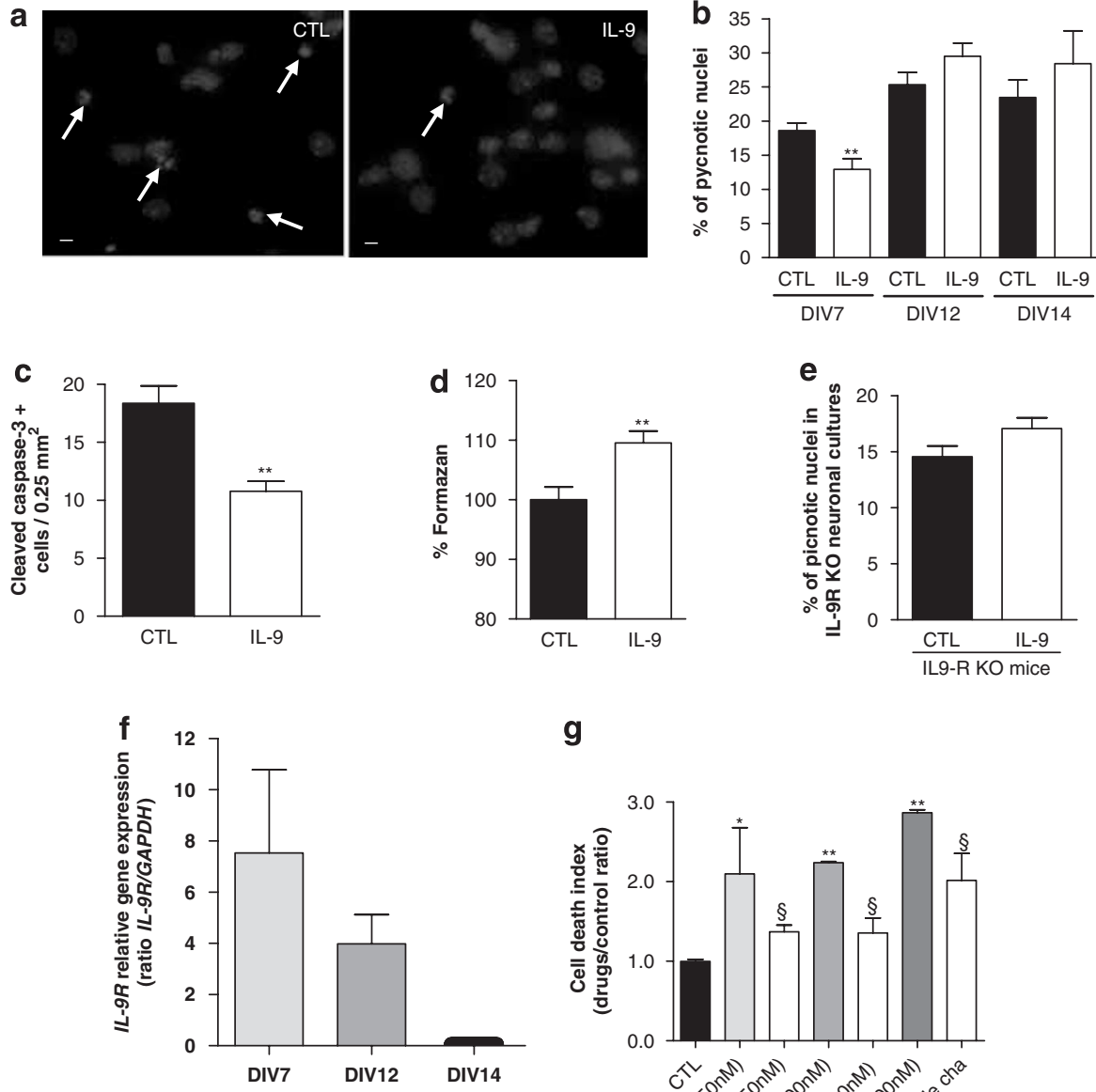

g

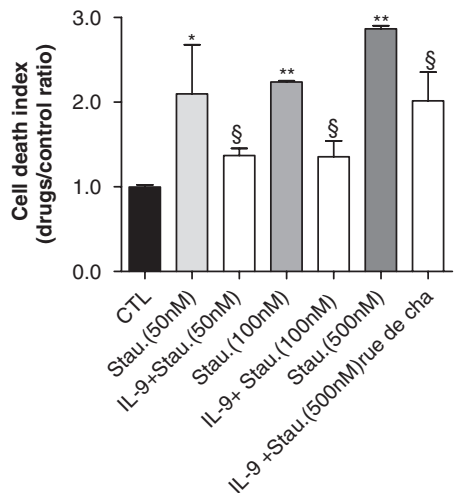

Figure 4 Primary neuron culture displays in vitro reduction in apoptosis when treated with IL-9. (a) Hoescht staining showed a drastic reduction in numbers of dying neurons when cultured in the presence of $200 \mathrm{U}$ of IL-9 (IL-9) from DIV 5 to DIV 7 (arrows point to picnotic nuclei) compared to neurons cultured with PBS (CTL). Scale $\mathrm{bar}=10 \mu \mathrm{m}$. (b) At DIV 7, dying cells (with picnotic nuclei) are significantly reduced in IL-9-treated cultures, compared to controls and compared to IL-9-treated cultures at DIV 12 and DIV 14. (c) Cell death reduction can be confirmed using cleaved caspase-3 marker by immunocytochemistry. Quantification showed a $40 \%$ reduction in activated caspase-3-positive cells in IL-9-treated cells at DIV 7, when compared to PBS-treated controls. (d) This result was confirmed using the MTS/Formazan. Treatment of neurons with 200 U IL-9 from DIV 5 to DIV 7 increases the cell viability. (e) At DIV 7, IL-9 treatment of IL-9R KO neurons did not induce any significant effect on cell death when compared to untreated IL-9 KO mice neurons. (f) This time-dependent loss of IL-9 effects correlated with the time-course reduction in IL-9R expression as shown in quantitative real-time PCR. (g) Experimentally induced neuronal cell death by staurosporin (stau) can be efficiently blocked by cotreatment with 200 U IL-9 from DIV 5 to DIV 7 . Each experiment was run twice using independent cultures, and in both cases, samples were assessed in triplicate. One-way ANOVA followed by post hoc test (time-course and dose-dependent experiments) or $T$-test (CTL versus IL-9) showed significant differences at ${ }^{\star} P<0.05,{ }^{* \star} P<0.01$. ${ }^{\S}$ Statically significant differences between staurosporine-treated cells and staurosporine + IL-9 cotreated cells $\left({ }^{\S} P<0.05\right)$ 
a

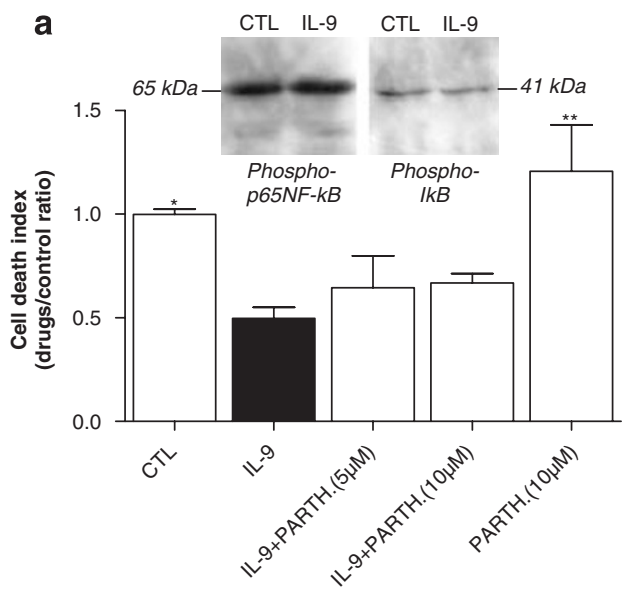

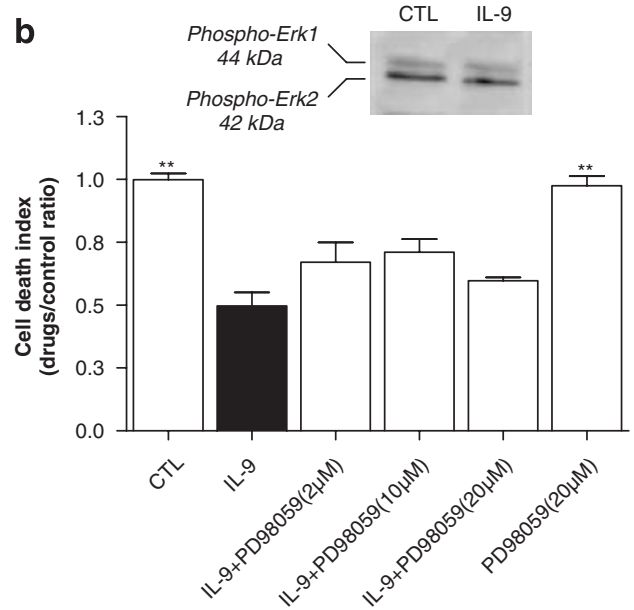

e
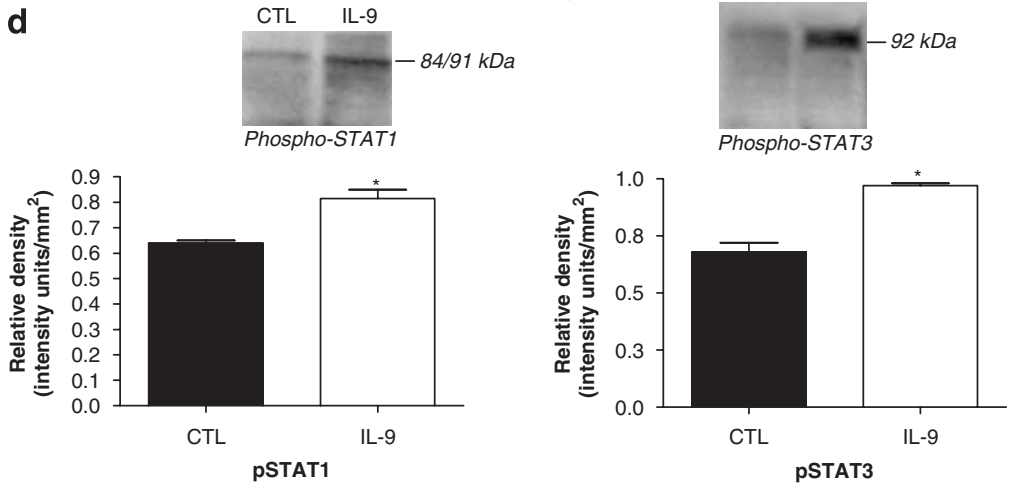

CTL IL-9

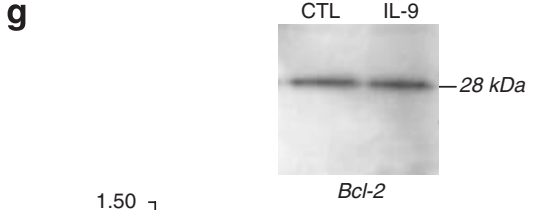

h

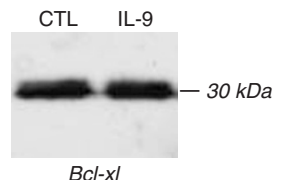

$\mathbf{f}$

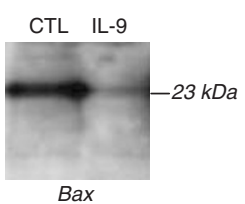

g

IL-9

Bax

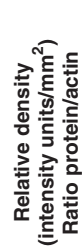

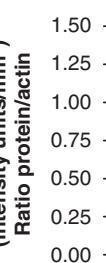

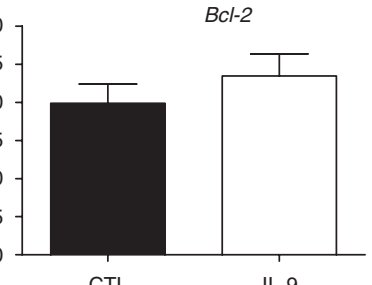

Bcl-2

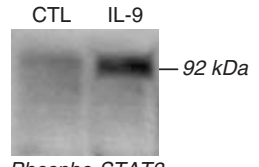

PSTAT3

Figure 5 Signaling pathway mediating antiapoptotic effects of IL-9 on primary neuronal cultures. (a-c) Selective inhibition of IL-9-induced apoptosis by a panel of selective pharmacological inhibitors was assessed using Hoechst staining. Treatments with parthenolide (Parth., a), PD98059 (b) and AG490 (c) were coupled with IL-9 treatment to selectively target NF- $\kappa \mathrm{B} / / \kappa \mathrm{B}$, Erk $1 / 2$ and JAK/STAT signaling cascades. Although treatment with $\mathrm{AG} 490$ greatly reduced either basal or IL-9 effects, NF- $\kappa \mathrm{B} / / \kappa \mathrm{B}$ and Erk inhibitors did not affect IL-9 effects on neuronal cell death. The latter pathways were not significantly activated by IL-9 treatment as shown by western blotting targeting phosphorylated forms of NF- $\kappa \mathrm{B} / 1 \kappa \mathrm{B}$ (insets in a) or phospho-Erk1/2 (insets in $\mathbf{b}$ ). Each experiment was run in duplicate. Asterisks indicate statistically significant differences between IL-9-treated cells and control (CTL: PBS or DMSO) or cells treated with inhibitors ( ${ }^{\star} P<0.05,{ }^{\star \star} P<0.01$ in ANOVA followed by post hoc test). (d and e) Western blot analysis and corresponding quantification of selective components of STAT-mediated apoptotic pathway using proteins extracted from neurons at 7 DIV treated with IL-9 or PBS. Levels of phospho-STAT proteins 1 and 3 were increased by IL-9 treatment when compared to PBS-treated cells. (f-h) Pro-apoptotic mitochondrial factor Bax was found decreased by IL-9 treatment as showed with western blot analysis and corresponding quantification. Conversely, expression of the antiapoptotic factors Bcl-2 ( $\mathbf{g})$ and Bcl-xl (h) were not affected by IL-9 treatment. Asterisks indicate statistically significant differences between PBS-treated controls and wells treated with IL-9 for both ages $\left({ }^{*} P<0.05\right.$ in a T-test) 
also shown to modulate T-cell and mast-cell survival by inducing $B C L-3$ gene, which encodes a protein member of the $\mathrm{I} \kappa \mathrm{B}$ family, ${ }^{20} \mathrm{NF}-\kappa \mathrm{B} / \mathrm{I}-\kappa \mathrm{B}$ pathway did not seem to be critically involved in IL- 9 effects on cultured neurons. In addition, the Erk pathway, which is involved in IL-9 effects on T cells ${ }^{17}$ and which is known to mediate the effects of several trophic factors such as BDNF, ${ }^{28}$ did not seem to be significantly involved in the present study. Together, these data show both similarities in and differences between immune cells and neurons with respect to transduction mechanisms involved in IL-9 antiapoptotic effects.

Cell death partly depends on the ratio of pro-apoptotic/ antiapoptotic factors expressed by mitochondria. Further supporting the antiapoptotic role of IL-9 for developing neurons, the present study showed that IL-9 significantly inhibited the expression of Bax, a pro-apoptotic molecule in newborn rodents, ${ }^{3}$ whereas it had no detectable effect on the antiapoptotic protein $\mathrm{Bcl}-2$ and $\mathrm{Bcl}-\mathrm{xl}$. The present result is in agreement with the literature showing that the sensitivity of neurons to apoptosis-inducing signals may be modulated by Bax levels and that Bax-induced cell death requires caspase activation. ${ }^{13}$ However, IL-9 effects on the other members of the $\mathrm{Bcl}$ and Bax protein families such as Bim and Bak (which may participate in the compensatory mechanism triggered in the absence of $\mathrm{Bax}^{29}$ ), Alix (which is a protein regulating endosomal trafficking and implicated in neuronal death ${ }^{30}$ ) or PUMA (the overexpression of which is sufficient to induce Bax-dependent cortical neuron death ${ }^{31}$ ) remain to be further investigated in neurons. Also, the precise interactions between the JAK/STAT pathway and these pro- and antiapoptotic factors remain unclear.

As discussed above, IL-9/IL-9R antiapoptotic effect seems to affect predominantly superficial cortical layers (layers II-III). Interestingly, inhibition of the NMDA receptors or excessive activation of the GABA-A receptors significantly enhance neuronal cell death in layers II and V of the rodent neocortex, ${ }^{32}$ suggesting that different mechanisms could control developmental apoptosis of neurons located in different neocortical layers.

Despite the fact that apoptosis as measured by cleaved caspase-3 in P5 neocortex is almost doubled in IL-9R KO when compared to wild-type animals, we did not observe any significant change in neocortical neuronal density in adult KO animals. This apparent discrepancy could be linked to different factors. First, we measured neuronal density and not the total number of neocortical neurons. Indeed, a reduction in neuronal number is not necessarily associated with a significant change in neuronal density. ${ }^{33}$ In addition, previous studies have suggested that about 10\% (at most $15 \%$ ) of all neocortical neurons undergo apoptosis during the P0-P14 interval, ${ }^{4}$ suggesting that IL-9R KO animals would have about $20 \%$ (at most $30 \%$ ) of postnatal neuronal apoptosis. If this is true, adult ILK-9R KO would have a predicted reduction of about $11 \%$ (at most $18 \%$ ) of the total number of neocortical neurons when compared to controls. Such a reduction could remain unnoticed in terms of brain size without a detailed analysis based on stereological approaches. Finally, it is likely that several factors are controlling the cell death and survival of a given population of neocortical neurons during early postnatal life in mice. ${ }^{34} \mathrm{~A}$ good example of this redundancy is the fact that several $\mathrm{KO}$ mice with genetic deletion of members of the Bax/Bcl-2 family do not show any detectable neocortical phenotype. ${ }^{35}$ Accordingly, one may expect that, despite the increased apoptosis of neurons in P5 IL-9R KO mice, other antiapoptotic factors might counteract this negative effect during the last phase of PCD.

Altogether, the present results strongly support the original hypothesis that the IL-9/IL-9R signaling pathway, which is specifically expressed in a time-restricted manner during the peak of neocortical PCD, represents a novel endogenous antiapoptotic mechanism. The selective pattern of expression of IL-9R mRNA suggests that this IL-9/IL-9R pathway protects a subset of neurons against PCD.

\section{Materials and Methods}

Animals. Swiss mice were obtained from Janvier (Elevage Janvier, Le GenestSt-Isle, France). As previously described ${ }^{36}$ IL-9R KO mice were obtained in the C57BL6 background by replacing a 2.8-kb genomic Kpnl-Spel fragment containing exons $2-6$ by a neomycin resistance gene, leading to a complete loss of the extracellular binding domain.

IL-9 pretreatment. Mouse recombinant IL-9 (Endogen, Woburn, MA, USA) at a dose of $10 \mu \mathrm{g} / \mathrm{kg}$ diluted in $5 \mu \mathrm{l}$ PBS (phosphate buffer saline) was injected i.p. twice a day (between 0800 and 0010 hours and between 1800 and 2000 hours) on days P1-P4 and once a day (between 0800 and 0010 hours) on P5 in Swiss and IL-9R KO mice. Both sham- and IL-9-treated pups were killed at P5 (between 1800 and 2000 hours).

Quantitative real-time PCR. DNA-free total RNA from brain cortex, thymus or cell cultures were obtained using an adapted protocol from original publication. ${ }^{37}$ RNA quality was assessed using Experion bioanalyzer (Bio-Rad, Hercules, CA, USA). Primers were designed using M-fold and Oligo6 softwares based on the published CDNA sequences encoding the mouse IL-9, IL-9R, $\gamma$-chain, mMCP-1 and TCR- $\beta$ genes. To standardize the gene expression across samples, we used the mouse HPRT (hypoxanthine-guanine phosphoribosyl-transferase) gene. For reverse transcription, we used the Iscript CDNA synthesis kit (Bio-Rad). Real-time PCR was set up using sybergreen-containing supermix (Bio-Rad). Each experiment was run twice, and in both cases, samples were assessed in triplicate.

In situ hybridization. Coronal sections $(10 \mu \mathrm{m})$ from P1, P5 and P20 Swiss mice were cut on a cryostat. cDNAs (clone p9RC4) encoding murine IL-9R ${ }^{18}$ were used as templates, and DIG-labeled (Roche Diagnostics, Basel, Switzerland) antisense RNA probes were made by in vitro transcription. In situ hybridization was performed as previously described. ${ }^{38}$

Immunohistochemistry. For caspase-3 detection, P5 pup brains from wildtype and IL-9R KO embedded in paraffin were cut coronally from the frontal to the occipital pole (10- $\mu \mathrm{m}$ sections). Sections were incubated with rabbit-cleaved caspase-3 antibody at a 1/200 dilution (Cell Signaling Technology, Danvers, MA, USA). For NeuN detection (dilution 1/400; Chemicon Guyancourt, France), adult brains from wild-type and IL-9R KO embedded in paraffin were cut sagittally $(10 \mu \mathrm{m}$ sections). Detection of labeled antigens was performed with avidin-biotin horseradish peroxidase kits (Vector, Burlingame, CA, USA) according to instructions. Adjacent sections were used as negative controls in which the incubation with primary antibody was omitted. Two investigators, both of whom were blinded with respect to treatment group, estimated independently the number of positive cells, focusing on the motor, somatosensory and visual cortex. Six animals were included in each experimental group. For each animal and each cortical area, two non-adjacent sections were immunoreacted in successive experiments.

For double immunostaining, P5 Swiss mice were transcardially perfused with 4\% paraformaldehyde under anesthesia with inhaled isoflurane (Abbott France, Rungis, France). The brains were postfixed in the same fixative for $3 \mathrm{~h}$, cryoprotected in sucrose and frozen in liquid nitrogen-cooled isopentane. Coronal sections, $10 \mu \mathrm{m}$ thick, were serially cut. The sections were incubated overnight with primary anticleaved caspase-3 antibody in association with one of the following antibody: anti-GFAP (Sigma, Lyon, France; at a dilution of 1/500), anti-somatostatin (Santa 
Cruz Biotechnology, Santa Cruz, CA, USA; at 1/1000) or anti-Brn-1 (Santa Cruz Biotechnology; at 1/500). Detection of labeled antigens was performed with a Cy3-conjugated antibody (red color; Jackson Immunoresearch, Suffolk, UK) for cleaved caspase-3 and Fluoprobe 488-conjugated antibody (green color; Interchim, Montluçon, France) for neural markers.

TUNEL staining. Sections adjacent to those used for immunohistochemistry were treated with proteinase $\mathrm{K}$ for $20 \mathrm{~min}$ at $37^{\circ} \mathrm{C}$, then permeabilized in a $0.1 \%$ solution of Triton X-100 in $0.1 \mathrm{M}$ sodium citrate solution for $2 \mathrm{~min}$ at $4^{\circ} \mathrm{C}$. TUNEL staining was made according to the manufacturer's instructions (Roche Diagnostics).

Cell cultures. Primary neuronal cultures ( $>98 \%$ purity assessed by MAP-2 (microtubule-associated protein-2) immunostaining, data not shown) were prepared from E14.5 embryonic mice. ${ }^{26}$ For oligodendrocyte progenitors, astrocytes and microglia primary cultures, cell populations were isolated from P0 and P2 newborn brains, respectively, and subsequently cultured according to published protocols for 3 weeks. ${ }^{26,39,40}$ For mast cell cultures, the D-36 murine mast cell line was used. ${ }^{26}$

The neurons were maintained for 7-14 days and pretreated with $200 \mathrm{U}$ $(20 \mathrm{ng} / \mathrm{ml}) \mathrm{IL}-9$ for $48 \mathrm{~h}$ to ensure a maximum IL- 9 cell-death inhibition.

For signal transduction studies, neurons treated with $200 \mathrm{U}$ IL-9 during $48 \mathrm{~h}$ between DIV 5 and DIV 7 were also treated with specified concentrations of various inhibitors of protein kinases (staurosporine and PD98059 diluted in DMSO; Sigma), $\mathrm{NF}-\kappa \mathrm{B}$ (parthenolide diluted in DMSO; Sigma) or JAK/STAT pathways (AG 490 diluted in PBS; Tocris, Bristol, UK). Cell death was measured using Hoechst at a concentration of $2 \mathrm{mg} / \mathrm{l}$ and cell viability was monitored using the MTS/Formazan assay (CellTiter 96 Aqueous One Solution Cell Proliferation Assay; Promega, Charbonnieres, France). As positive controls of the efficacy of parthenolide and PD98059 in our experimental paradigm, neurons were treated with CNTF $(5 \mathrm{nM}$; Sigma), CNTF $(5 \mathrm{nM})+$ parthenolide $(10 \mu \mathrm{M})$, BDNF $(10 \mu \mathrm{M})$, BDNF $(10 \mu \mathrm{M})+$ PD98059 $(5 \mu \mathrm{M})$ in similar conditions. On western blots, CNTF induced a significant increase in the levels of phosphorylated forms of NF- $\kappa B$ and this effect was abolished by cotreatment with parthenolide (data not shown). Similarly, BDNF induced a significant increase in the levels of phosphorylated Erk1/2 and this effect was abolished by cotreatment with PD98059 (data not shown)

Immunocytochemistry. For caspase-3 detection, DIV 7 cell cultures treated with PBS or $200 \mathrm{U}$ IL-9 for $48 \mathrm{~h}$ were reacted with rabbit-cleaved caspase-3 antibody at a 1/500 dilution (Cell Signaling Technology). Detection of labeled antigens was performed with a Cy3-conjugated donkey anti-rabbit antibody (Jackson Immunoresearch). Two investigators, both of whom were blinded with respect to treatment group, independently estimated the number of positive cells.

Western blot analysis. Total proteins were extracted from neuronal cultures according to the protocol of a commercially available kit (Cell Signaling Technology). A panel of different antibodies was used including STAT1 (Cell Signaling Technology; 1/100), STAT3 (Santa Cruz Biotechnology; 1/200), phospho-STAT1/3 (pSTAT1/3, Santa Cruz Biotechnology; 1/100), Bax (Santa Cruz Biotechnology; 1/250), Bcl-2 (Santa Cruz Biotechnology; 1/250), Bcl-xl (Santa Cruz Biotechnology; 1/100), phospho-p65 NF- $\kappa$ B (Cell Signaling Technology; 1/1000), phospho-l $\kappa \mathrm{B}-\alpha$ (Cell Signaling; 1/1000) and phospho-Erk (pErk1/2, Sigma; $1 / 4000$ ). To standardize the protein expression across samples, we used an anti- $\beta$-actin goat antibody (Santa Cruz Biotechnology) at a concentration of 1/5000. Western blot experiments were run in duplicate. Differences in protein concentrations between samples were estimated using Quantity One software from Biorad.

Statistical analysis. The data were analyzed with a Student's $t$-test or a oneway ANOVA (analysis of variance). In ANOVA analyses, when a main effect was found to be significant, post hoc multiple comparison tests were performed (Bonferroni, Dunnett or Kruskal-Wallis; Graph-Pad Prism version 4.01 for Windows, Graph-Pad Software, San Diego, CA, USA).

Acknowledgements. We thank Leslie Schwendimann, Danielle Rouellé and Véronique Massonneau for excellent technical assistance. This work was financially supported by Inserm and the Universite Paris 7. RF was a recipient of graduate studen fellowship from national foundations including APETREIMC, CDI, SESEP and FRM. VD was financially supported by a graduate student fellowship from the Fondation Motrice. VL was financially supported by a postdoctoral fellowship from FRM.
1. Oppenheim RW. Cell death during development of the nervous system. Annu Rev Neurosci 1991; 14: 453-501.

2. Ferrer I, Soriano E, del Rio JA, Alcantara S, Auladell C. Cell death and removal in the cerebral cortex during development. Prog Neurobiol 1992; 39: 1-43.

3. Stankovski L, Alvarez C, Ouimet T, Vitalis T, El-Hachimi KH, Price D et al. Developmental cell death is enhanced in the cerebral cortex of mice lacking the brain vesicular monoamine transporter. J Neurosci 2007; 27: 1315-1324.

4. Verney C, Takahashi T, Bhide PG, Nowakowski RS, Caviness Jr VS. Independent controls for neocortical neuron production and histogenetic cell death. Dev Neurosci 2000; 22: 125-138.

5. McFarland KN, Wilkes SR, Koss SE, Ravichandran KS, Mandell JW. Neural-specific inactivation of ShcA results in increased embryonic neural progenitor apoptosis and microencephaly. J Neurosci 2006; 26: 7885-7897.

6. Naruse I, Keino H. Apoptosis in the developing CNS. Prog Neurobiol 1995; 47: 135-155

7. Sulik KK, Cook CS, Webster WS. Teratogens and craniofacial malformations: relationships to cell death. Development 1988; 103 (Suppl): 213-231.

8. Dikranian K, Ishimaru MJ, Tenkova T, Labruyere J, Qin YQ, Ikonomidou C et al. Apoptosis in the in vivo mammalian forebrain. Neurobiol Dis 2001; 8: 359-379.

9. Adams JM, Cory S. The Bcl-2 protein family: arbiters of cell survival. Science 1998; 281: $1322-1326$

10. Kuida K, Haydar TF, Kuan CY, Gu Y, Taya C, Karasuyama H et al. Reduced apoptosis and cytochrome c-mediated caspase activation in mice lacking caspase 9. Cell 1998; 94: 325-337.

11. Pompeiano M, Blaschke AJ, Flavell RA, Srinivasan A, Chun J. Decreased apoptosis in proliferative and postmitotic regions of the Caspase 3-deficient embryonic central nervous system. J Comp Neurol 2000; 423: 1-12.

12. Oltvai ZN, Milliman CL, Korsmeyer SJ. Bcl-2 heterodimerizes in vivo with a conserved homolog, Bax, that accelerates programmed cell death. Cell 1993; 74: 609-619.

13. Vekrellis K, McCarthy MJ, Watson A, Whitfield J, Rubin LL, Ham J. Bax promotes neuronal cell death and is downregulated during the development of the nervous system. Development 1997; 124: 1239-1249.

14. Sun W, Winseck A, Vinsant S, Park OH, Kim H, Oppenheim RW. Programmed cell death of adult-generated hippocampal neurons is mediated by the proapoptotic gene Bax. J Neurosci 2004; 24: 11205-11213.

15. Alcantara S, Frisen J, del Rio JA, Soriano E, Barbacid M, Silos-Santiago I. TrkB signaling is required for postnatal survival of CNS neurons and protects hippocampal and motor neurons from axotomy-induced cell death. J Neurosci 1997; 17: 3623-3633.

16. Arsenijevic $Y$, Weiss $S$. Insulin-like growth factor-I is a differentiation factor for postmitotic CNS stem cell-derived neuronal precursors: distinct actions from those of brain-derived neurotrophic factor. J Neurosci 1998; 18: 2118-2128.

17. Knoops L, Renauld JC. IL-9 and its receptor: from signal transduction to tumorigenesis. Growth Factors 2004; 22: 207-215

18. Renauld JC, Druez C, Kermouni A, Houssiau F, Uyttenhove C, Van Roost E et al. Expression cloning of the murine and human interleukin 9 receptor cDNAs. Proc Natl Acad Sci USA 1992; 89: 5690-5694.

19. Benczik M, Gaffen SL. The interleukin (IL)-2 family cytokines: survival and proliferation signaling pathways in T lymphocytes. Immunol Invest 2004; 33: 109-142.

20. Richard M, Louahed J, Demoulin JB, Renauld JC. Interleukin-9 regulates NF-kappaB activity through BCL3 gene induction. Blood 1999; 93: 4318-4327.

21. Renauld JC, Vink A, Louahed J, Van Snick J. Interleukin-9 is a major anti-apoptotic factor for thymic lymphomas. Blood 1995; 85: 1300-1305.

22. Mehler MF, Rozental R, Dougherty M, Spray DC, Kessler JA. Cytokine regulation of neuronal differentiation of hippocampal progenitor cells. Nature 1993; 362 : $62-65$.

23. Lee YB, Nagai A, Kim SU. Cytokines, chemokines, and cytokine receptors in human microglia. J Neurosci Res 2002; 69: 94-103.

24. Benveniste EN. Cytokine actions in the central nervous system. Cytokine Growth Factor Rev 1998; 9: 259-275.

25. Sepulveda $P$, Encabo A, Carbonell-Uberos F, Minana MD. BCL-2 expression is mainly regulated by JAK/STAT3 pathway in human CD34(+) hematopoietic cells. Cell Death Differ 2007; 14: 378-380.

26. Mesples B, Fontaine RH, Lelievre V, Launay JM, Gressens P. Neuronal TGF-beta1 mediates IL-9/mast cell interaction and exacerbates excitotoxicity in newborn mice. Neurobiol Dis 2005; 18: 193-205.

27. Bauer JH, Liu KD, You Y, Lai SY, Goldsmith MA. Heteromerization of the gamma c chain with the interleukin-9 receptor alpha subunit leads to STAT activation and prevention of apoptosis. J Biol Chem 1998; 273: 9255-9260.

28. Huang EJ, Reichardt LF. Trk receptors: roles in neuronal signal transduction. Annu Rev Biochem 2003; 72: 609-642.

29. Gavalda N, Perez-Navarro E, Garcia-Martinez JM, Marco S, Benito A, Alberch J. Bax deficiency promotes an up-regulation of $\operatorname{Bim}(E L)$ and Bak during striatal and cortical postnatal development, and after excitotoxic injury. Mol Cell Neurosci 2008; 13 . 331-335. 
30. Trioulier Y, Torch S, Blot B, Cristina N, Chatellard-Causse C, Verna JM et al. Alix, a protein regulating endosomal trafficking, is involved in neuronal death. $J$ Biol Chem 2004; 279: 2046-2052.

31. Uo T, Kinoshita $Y$, Morrison RS. Apoptotic actions of $p 53$ require transcriptional activation of PUMA and do not involve a direct mitochondrial/cytoplasmic site of action in postnatal cortical neurons. J Neurosci 2007; 27: 12198-12210.

32. Ikonomidou C, Bittigau P, Koch C, Genz K, Hoerster F, Felderhoff-Mueser U et al. Neurotransmitters and apoptosis in the developing brain. Biochem Pharmacol 2001; 62: 401-405.

33. Gressens P, Marret S, Hill JM, Brenneman DE, Gozes I, Fridkin M et al. Vasoactive intestinal peptide prevents excitotoxic cell death in the murine developing brain. $J$ Clin Invest 1997; 100: 390-397.

34. Kuan CY, Roth KA, Flavell RA, Rakic P. Mechanisms of programmed cell death in the developing brain. Trends Neurosci 2000; 23: 291-297.
35. Roset R, Ortet L, Gil-Gomez G. Role of Bcl-2 family members on apoptosis: what we have learned from knock-out mice. Front Biosci 2007; 12: 4722-4730.

36. Steenwinckel V, Louahed J, Orabona C, Huaux F, Warnier G, McKenzie A et al. IL-13 mediates in vivo IL-9 activities on lung epithelial cells but not on hematopoietic cells. J Immunol 2007; 178: 3244-3251.

37. Chomczynski $P$, Sacchi N. Single-step method of RNA isolation by acid guanidinium thiocyanate-phenol-chloroform extraction. Anal Biochem 1987; 162: 156-159.

38. Marillat V, Cases O, Nguyen-Ba-Charvet KT, Tessier-Lavigne M, Sotelo C, Chedotal A. Spatiotemporal expression patterns of slit and robo genes in the rat brain. J Comp Neurol 2002; 442: 130-155.

39. McCarthy KD, de Vellis J. Preparation of separate astroglial and oligodendroglial cell cultures from rat cerebral tissue. J Cell Biol 1980; 85: 890-902.

40. Hetier E, Ayala J, Denefle P, Bousseau A, Rouget P, Mallat M et al. Brain macrophages synthesize interleukin-1 and interleukin-1 mRNAs in vitro. J Neurosci Res 1988; 21: 391-397.

Supplementary Information accompanies the paper on Cell Death and Differentiation website (http://www.nature.com/cdd) 